Article

\title{
Analysis of Spectrum Sensing over Imperfect Channel conditions in Cognitive Vehicular Networks
}

\author{
Xiaomin Qian ${ }^{1}$ (D), Li Hao ${ }^{1, *}$, Dadong $\mathrm{Ni}^{1}$ and Quang Thanh $\operatorname{Tran}^{1,2}$ \\ 1 Key Lab of Information Coding and Transmission, Southwest Jiaotong University, Chengdu 610031, China; \\ qxflying2009@163.com; lhao@swjtu.edu.cn; dadongni@hotmail.com \\ 2 Faculty of Electrical and Electronic Engineering, University of Transport and Communications, Hanoi, \\ Vietnam; thanhktvt@gmail.com \\ * Correspondence: qxflying2009@163.com; Tel.: +86-180-3966-2390
}

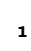

2
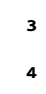

\begin{abstract}
An explosive growth in vehicular wireless services and applications gives rise to spectrum resource starvation. Cognitive radio has been used to vehicular networks to mitigate the impending spectrum starvation problem by allowing vehicles to fully exploit spectrum opportunities unoccupied by licensed users. Efficient and effective detection of licensed user is a critical issue to realize cognitive radio applications. However, spectrum sensing in vehicular environments is a very challenging task due to vehicles mobility. For instance, vehicle mobility has a large effect on the wireless channel, thereby impacting the detection performance of spectrum sensing. Thus, gargantuan efforts have been made in order to analyze the fading properties of mobile radio channel in vehicular environments. Indeed, numerous studies have demonstrated that the wireless channel in vehicular environments can be characterized by a temporally correlated Rayleigh fading. In this paper, we focus on energy detection for spectrum sensing and a counting rule for cooperative sensing based on Neyman-Pearson criteria. Further, we go into the effect of the sensing and reporting channels condition on spectrum sensing performance under temporally correlated Rayleigh sensing channel. For local and cooperative sensing, we derive some alternative expressions for average probability of miss detection. The pertinent numerical and simulating results are provided to further validate our theoretical analyses under a variety of scenarios.
\end{abstract}

Keywords: Cognitive radio, Cognitive vehicular networks, Spectrum sensing, Sensing/Reporting channel, Correlated Rayleigh fading channel, Hard fusion.

\section{Introduction}

Vehicular networks serve as an actual enabling application that is conceived to enhance road safety and provide in-vehicle infotainment for passengers on the road by allowing vehicle-to-vehicle (V2V) as well as vehicle-to-infrastructure (V2I) communications. It has attracted substantial investigation from industries and research institutions in the last few years and still faces a host of challenges before starting the actual implementation. One major challenge is the deficient frequency resources available for wireless communications in vehicular networks.

Currently, the Federal Communication Commission (FCC) has reserved 75-MHz licensed spectrum bandwidth (i.e., seven $10-\mathrm{MHz}$ channels) at a center frequency of $5.9-\mathrm{GHz}$ in support of dedicated short-range communication (DSRC) in vehicular environments. However, a tremendous increase in wireless applications being developed for vehicular communications, may give rise to serious congestion of the band, and ultimately reducing the communication efficient for safety applications. Moreover, not only road safety applications, but also the increasing number of 
applications related to infotainment systems will also lead to channel contention and spectrum deficiency. In view of the stringent QoS requirements on DSRC spectrum, it is not be sufficient for all applications to depend only on the 5.9-GHz DSRC spectrum. There is a dramatic increase in the demand for frequency resources to satisfy their communication requirements.

To relieve the bottleneck problem of spectrum deficiency, cognitive radio (CR) [1], [2] has increasingly been presented as a potential technique being capable of accessing licensed but unoccupied frequency bands only without causing any unacceptable interference to licensed users (or primary users, PUs). Cognitive radio has become one of the most breathtaking technologies to improve the spectrum efficiency effectively for the past couple of decades. In addition to a better remedy for frequency scarcity issue, cognitive radio is appropriate for vehicular environments, since their unique characteristics make it much better to achieve the spatial and temporal reusage of the empty frequency bands of PUs compared to other traditional cognitive networks [3], [4]. To efficiently reutilize the spectrum holes with minimum interference to PUs, the CR-enabled vehicles, which can be called secondary vehicular users (SVUs), need to reliably make a decision about the existence of PUs. And they must immediately vacate the radio channels as soon as PUs are detected. Consequently, spectrum sensing constitutes the key component of cognitive vehicular networks (CVNs) [7].

Spectrum sensing [5] is a wealthily investigated subject, but is more concentrated in traditional CR networks. Among them, cooperative sensing [6] has already attracted strong research interest as it can take further advantage of both spatial and temporal diversity, and thereby garner better sensing accuracy and efficiency in the face of fading. However, these existing spectrum sensing techniques can not be directly applicable to vehicular networks. And it's all because we have to give an account of the idiosyncrasies of vehicular networks such as high mobility while designing sensing schemes for CVNs [3], [8]. For instance, rapid movement of vehicles makes the availability of spectrum holes dynamically change since a vehicle may enter or leave a region interfered by a particular PU at different locations along the road. In this regard, it is considerable for SVUs to detect PU activities in the fastest possible way. Additionally, the vehicles' motion are restricted and predictable due to the fixed road topology. In consequence, each vehicle may be glad to know in advance the spectrum opportunities to better utilize them for transmission on its track. Further, high speeds and the environmental clutter can affect the received signal due to Doppler effect, fading and shadowing. These factors will have immediate impacts on spectrum sensing of CVNs.

Spectrum sensing in dynamic vehicular environments have been researched in some preliminary works [4], [9], [10], [11], [12]. The authors in [4] proposed a novel adaptive sensing coordinated mechanism, in which the central node merely assist and coordinate the SVUs to better acquire the availability of spectrum holes instead of completely controlling the sensing and access. The authors in [9] studied the detection performance of spectrum sensing under the shadowing and multi-path composite fading channel in vehicular environments. The authors in [10] presented an asynchronous collaborative sensing framework in which the tagged vehicle collects energy measurements labeled with time and location information from collaborative SVUs and assigns weights based on their storing time and location. The authors in [11] proposed a distributed collaborative sensing scheme based on adaptive decision threshold for sensing and voting scheme for connected vehicles. An integrated overview of spectrum sensing in cognitive vehicular environments can be discovered in [12]. None of these previous researches considered the effect of temporal correlation due to vehicle motion and multi-path propagation in a mobile vehicular environment. Moreover, the reporting channels between SVUs and the fusion center (FC) were assumed to be ideal.

In this paper, our attention is centered on large-scale fixed PUs detection in an infrastructure-based $\mathrm{CVN}$ based on mutual benefit and acquiring a win-win situation by allowing the back vehicles utilize the spectrum availability information after front vehicles sensed. Each SVU periodically performs sensing and reports its sensing information via the dedicated reporting channel to the nearby FC. The FC fuses the received information to make a global decision for the current cell. At this moment, some vehicles taking part in cooperative sensing may have left this cell. We investigate the sensing 
performance using hard fusion [13]. Although soft fusion can gather improved performance than hard fusion, the burden of reporting overhead impedes its applicability [14]. Our research in this paper distinct from the previous works in two respects: 1) look at the effect of fading correlation and their severity on spectrum sensing performance under temporally correlated Rayleigh sensing channel; and 2) make clear how dramatically the reporting channel conditions could influence the reliability of a local/global decision, when made by the FC. For local and cooperative sensing, we evaluate the sensing performances through theoretical analysis or Monte Carlo simulation. Our results shows that poor channel conditions harms the detection performance. However, amazingly, we will demonstrate that the fast time-varying characteristic of sensing channels can give us an opportunity to obtain temporal diversity provided that a proper sensing technique is adopted.

The rest of the paper is organized as follows. In Section 2, we describe system model and assumptions on spectrum sensing in vehicular environments. In Section 3, the local sensing performance over correlated Rayleigh fading is analyzed. In Section 4, we consider the cooperative sensing performance with fading reporting channels. This is followed by the numerical and simulated results in Section 5. Lastly, we summarize the conclusions in Section 6.

Throughout this paper on detection performance, we will declare the local probabilities of false alarm, miss detection, and detection as $P_{f}, P_{m}$, and $P_{d}$, respectively, whereas their equivalent local probabilities will be represented by $P_{F}, P_{M}$, and $P_{D}$ and their global probabilities $Q_{f}, Q_{m}$, and $Q_{d}$ for hard fusion.

\section{System Model and Problem Formulation}

In this section, we briefly introduce the network model, the channel model, the sensing model, and the assumptions made in the CVNs under consideration.

\subsection{Network Model}

We consider a vehicular network in multi-lane highway scenarios in which licensed and cognitive users coexisting peacefully within the same geospatial region and share multiple frequency channels, like the one depicted in Fig.1. We suppose a large-scale fixed licensed user, such as licensed DTV spectrum holders. The cognitive network is an infrastructure-aided network, in which each cell is composed of a fusion center and a number of associated SVUs. The FC coordinates SVUs collaborative sensing and their access to a vacant PU channel. Each SVU periodically performs sensing and reports its sensing information via the dedicated reporting channel to the nearby FC. According to the received local sensing results, the FC arrives at a global decision for the current cell. At this moment, some vehicles engaged in cooperative sensing may have left this cell. Hence, the FC diffuses the global decision to the next passing SVUs. We postulate that the vehicles move independently from any other, and thus, the sensing channels between PU and SVUs are all independent of one another. 


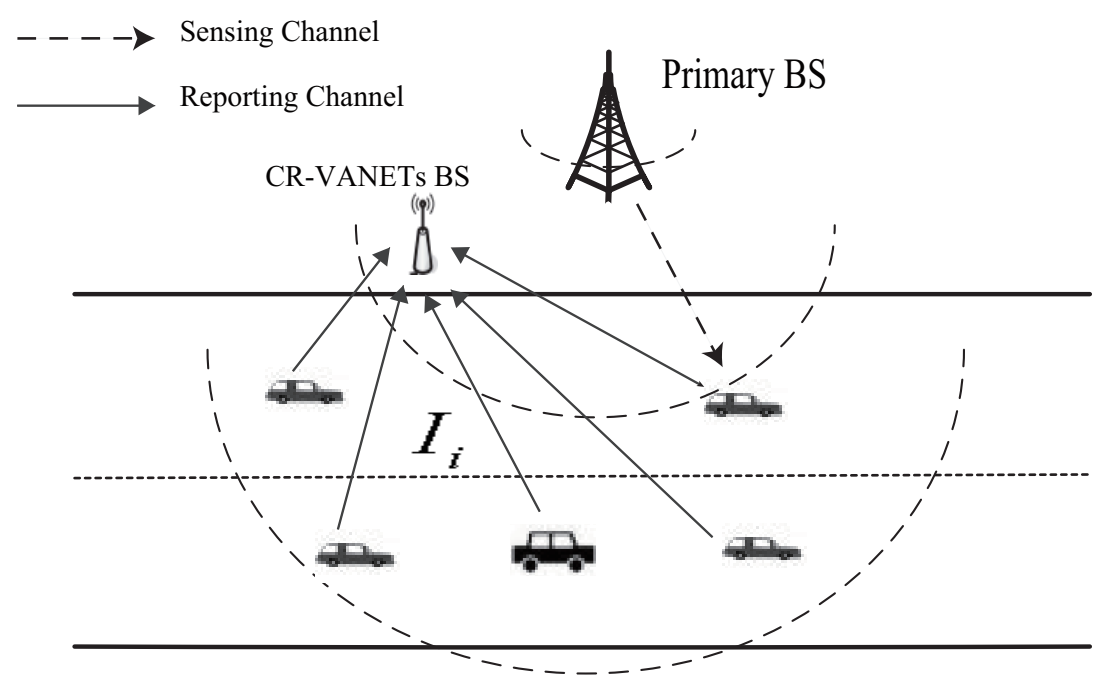

Figure 1. An illustrative example of cooperative sensing operation for cognitive vehicular networks. There are a PU, cooperative SVUs, and a CR-BS over wireless sensing/reporting channels.

116

\subsection{Channel Model}

In the above scenario, we consider the wireless channels, which have no correlation in space but are temporally correlated following the Jakes' model of land mobile fading channel (Table 2.1 in [15]). Let $\rho(\tau)$ denotes the correlation coefficient between two samples separating $\tau$ time interval, which satisfies

$$
\rho(\tau)=\mathcal{J}_{0}\left(2 \pi F_{d} T_{s} \tau\right)
$$

where $\mathcal{J}_{v}(\cdot)$ is defined by the $v$ th-order Bessel function of the first kind, and $F_{d} T_{s}$ represents normalized Doppler shift, $F_{d} T_{s}=\frac{f_{c} v}{c} T_{s}$, which may relate to a vehicle speed $v$, a carrier frequency $f_{c}$ and the sampling interval $T_{s}$.

The correlation specialities of the fading progress depend only on $F_{d} T_{s}$, as illustrated in fig. 2 . Based on the different $F_{d} T_{s}$, we can employ a suitable model, which is convenient to analyze the fading characteristics of sensing channel. For instance, three different models, $\mathcal{M}_{1}, \mathcal{M}_{2}$ and $\mathcal{M}_{3}$, are given as follows, respectively

$\mathcal{M}_{1}$ : When $F_{d} T_{s}$ is relatively smaller $(<0.001)$, the channel process is nearly time-invariant.

$\mathcal{M}_{2}$ : When $F_{d} T_{s}$ is small $(<0.02)$, the channel process is correlated ("slow" fading).

$\mathcal{M}_{3}$ : For larger values of $F_{d} T_{s}(>0.03)$, the channel process are almost independent time-varying ("fast" fading).

Note that $\mathcal{M}_{1}$ and $\mathcal{M}_{3}$ are two extreme cases of temporally correlated Rayleigh fading channel. 


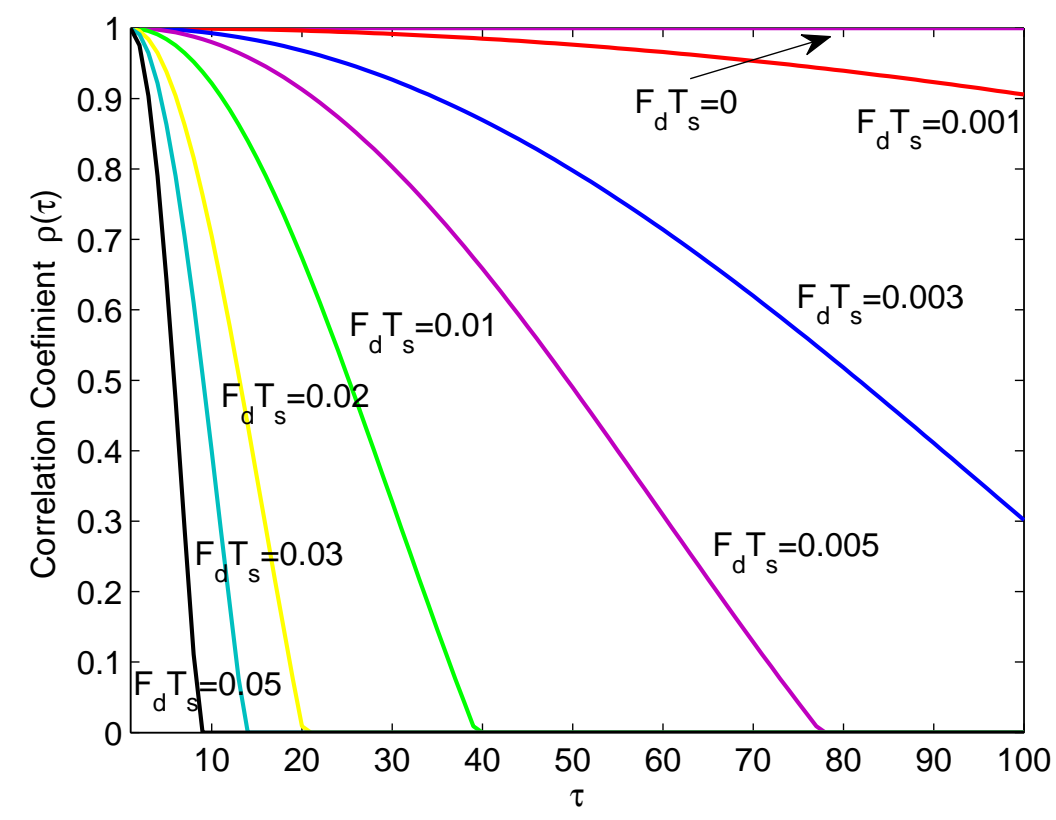

Figure 2. The correlation coefficient for various $F_{d} T_{s}$.

\subsection{Sensing Model}

Spectrum sensing is a binary hypothesis detection issue, with the null $\left(\mathcal{H}_{0}\right)$ and alternative $\left(\mathcal{H}_{0}\right)$ hypotheses associated to the presence and absence of PU in a given frequency band, respectively. We consider a cognitive vehicular network consisting of $N$ collaborative SVUs. Assume $L$ sampling observations can be used within a sensing interval. Under both hypotheses, the received observation by SUV $i$ at the $k$ th time instant can be expressed as, respectively

$$
x_{i}(k)= \begin{cases}n_{i}(k) & \mathcal{H}_{0} \\ h_{i}(k) s(k)+n_{i}(k) & \mathcal{H}_{1}\end{cases}
$$

where, $s(k)$ is the baseband-equivalent signal transmitted by the PU, $x_{i}(k)$ denotes the received signal by SVU $i, h_{i}(k)$ denotes the sensing channel gain with $\mathrm{E}\left[\left|h_{i}(k)\right|^{2}\right]=1$, and $n_{i}(k)$ denotes white and circularly symmetric complex Gaussian (CSCG) noise with mean zero and variance $\sigma_{n}^{2}$, i.e., $n_{i}(k) \sim \mathcal{C N}\left(0, \sigma_{n}^{2}\right)$. It's assumed that $s(k), h_{i}(k)$ and $n_{i}(k)$ are independent of each other, which is reasonable from a practical situation.

To reduce the complexity and reporting channel overhead, each SVU employs a mapping rule to its observations, to produce a quantized information denoted by $q\left(x_{i}\right)$. In this paper, we suppose each SUV makes a binary decision $u_{i}=q\left(x_{i}\right) \in\{+1,-1\}$ with probabilities of false-alarm and mis-detection $P_{f i}$ and $P_{m i}$. These decisions are then reported to the FC via a fading reporting channel or link, bit error may happen, which further affects the sensing performance at the FC. We will model the reporting channel as a Rayleigh fading channel. The received observation at the FC from the $i$ th node can be descried as

$$
z_{i}=g_{i} u_{i}+w_{i}
$$

where $g_{i}$ is the fading gain of reporting channel and $w_{i}$ is a zero-mean Gaussian random variable with variance $\delta_{i}^{2}$, i.e., $w_{i} \sim \mathcal{N}\left(0, \delta_{i}^{2}\right)$. Once the noisy observation $\left\{z_{i} ; i=1,2, \cdots, N\right\}$ is received and decoded, the FC makes a global decision on which hypothesis is more likely to be true. 
In the ordinary sense, an optimal fusion rule for hard combination is behaved in the form of a counting rule [16]. This argument proves to be true even when those decisions are received via unreliable communication channels, provided that the channels are also independent and identical distribution (IID). In the latter of this paper, we focus on energy detection for spectrum sensing and look at the detection performance of the counting rule in Rayleigh fading channels.

\section{Local Sensing with Energy Detection}

For local sensing, we consider energy detection (ED) instead of other sensing techniques, such as the cyclostationary feature detection which takes high computational complexity and much longer sensing time because it exploits a specific signature of the PU signal. Energy detection [17], [18] is the most practical and robust detection method because it merely estimates the signal energy on the considered band and produces very good performance without any priori information about PU signals, as illustrated in fig. 3. Furthermore, Energy detection is a viable choice for vehicular networks on account of its high mobile environment and low latency tolerant.

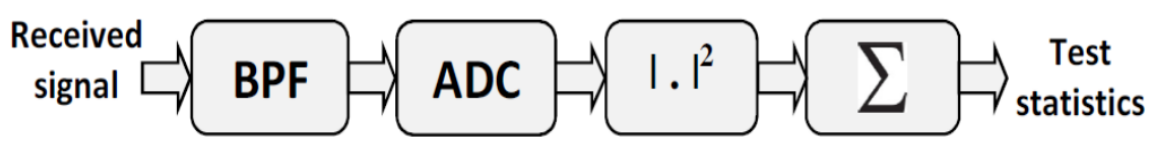

Figure 3. Energy detector model.

Consequently, the local test statistic of the energy detector at the $i$ th SUV, denoted by $e_{i}$, is basically an estimate of received signal energy which is represented as:

$$
e_{i}=\sum_{k=0}^{L-1}\left|x_{i}(k)\right|^{2}
$$

Let $\lambda$ denotes the local decision threshold and $u_{i}$ denote the local decision of the $i$ th SVU, the decision rule used by energy detector at each SVU can be expressed as

$$
u_{i}=\left\{\begin{array}{lll}
+1 & \text { if } \quad e_{i} \geq \lambda \\
-1 & \text { if } \quad e_{i}<\lambda
\end{array}\right.
$$

This means that when $e_{i}$ is greater than or equal to $\lambda$, SVU $i$ makes its individual decision $u_{i}=+1$ which tells PU signal is detected $\left(\mathcal{H}_{1}\right)$; otherwise, it makes a decision $u_{i}=-1$ which declares that PU signal is not detected $\left(\mathcal{H}_{0}\right)$.

For a high number of sensing observations $L$, on the basis of the Central Limit Theorem (CLT), the local test statistic $e_{i}$ given by (3) can be well modeled by a normal or Gaussian distribution under either $\mathcal{H}_{0}$ or $\mathcal{H}_{1}$ [17]. Accordingly, the probability density function (PDF) of $e_{i}$ at individual SVU can be compactly characterized by

$$
e_{i} \sim \begin{cases}\mathcal{N}\left(L \sigma_{n}^{2}, L \sigma_{n}^{4}\right) & \mathcal{H}_{0} \\ \mathcal{N}\left[\left(L+\eta_{i}\right) \sigma_{n}^{2},\left(L+2 \eta_{i}\right) \sigma_{n}^{4}\right] & \mathcal{H}_{1}\end{cases}
$$

where

$$
\eta_{i}=\sum_{k=0}^{L-1}\left|h_{i}(k)\right|^{2} \frac{E_{s}}{L \sigma_{n}^{2}}
$$

represents the instantaneous signal-to-noise ratio (SNR) experienced by the $i$-th SVU within the current sensing period. It is straightforward to see that $\eta_{i}$ varies from (sensing) period to period, since it 
generally hinges upon the fading characteristics of the wireless channel. And $E_{s}=\sum_{k=0}^{L-1}|s(k)|^{2}$ denotes the transmitted signal energy by the primary user over a sensing interval of $L$ sampling observations.

In the above discussed case, the probabilities of false alarm, miss detection and detection for local sensing at the $i$-th SVU can be represented by the following formulas, respectively

$$
\begin{gathered}
P_{f i}(\lambda)=P\left(e_{i} \geq \lambda \mid \mathcal{H}_{0}\right)=\mathcal{Q}\left(\frac{\lambda-L \sigma_{n}^{2}}{\sqrt{L} \sigma_{n}^{2}}\right) \\
P_{d i}(\lambda)=P\left(e_{i} \geq \lambda \mid \mathcal{H}_{1}\right)=\mathcal{Q}\left(\frac{\lambda-\left(L+\eta_{i}\right) \sigma_{n}^{2}}{\sqrt{L+2 \eta_{i}} \sigma_{n}^{2}}\right) \\
P_{m i}(\lambda)=P\left(e_{i}<\lambda \mid \mathcal{H}_{1}\right)=\mathcal{Q}\left(\frac{\left(L+\eta_{i}\right) \sigma_{n}^{2}-\lambda}{\sqrt{L+2 \eta_{i}} \sigma_{n}^{2}}\right)
\end{gathered}
$$

where $\mathcal{Q}(x)$ is defined by the complementary cumulative distribution function of the standard Gaussian, i.e., $\mathcal{Q}(x)=\frac{1}{\sqrt{2 \pi}} \int_{x}^{\infty} \exp \left(-\frac{t^{2}}{2}\right) d t$.

It's apparent that, $P_{f i}$ in (8) is independent of received SNR because $P_{f i}$ is considered for the hypothesis of no PU signal transmission. On the other hand, $P_{m i}$ in (10) is a conditional probability depending on instantaneous received SNR $\eta_{i}$. In this circumstance, the average $P_{m}$ can be calculated by integrating the conditional $P_{m i}$ over the SNR fading distribution [17], or quantify the quality of each channel by its corresponding average received SNR.

From (8) and (10), we also see that, $\lambda$ can be analytically set to maintain the desired $P_{f}$ if $P_{f}$ is designated as the constraint of the detection problem. Then, we obtain $P_{m i}$ related to the desired $P_{f}$ as follows

$$
P_{m i}\left(\eta_{i}\right)=\mathcal{Q}\left(\frac{\eta_{i}-\sqrt{L} \mathcal{Q}^{-1}\left(P_{f}\right)}{\sqrt{L+2 \eta_{i}}}\right)
$$

where $\mathcal{Q}^{-1}(x)$ is defined by the inverse function of $\mathcal{Q}(x)$.

Next, we first consider the case of $\mathcal{M}_{1}$, in which the vehicles move more slowly, $F_{d} T_{s}$ is small $(<0.001)$. And thus, the channel gain $h_{i}(k)$ remain virtually unchanged during the sensing interval, i.e., $h_{i}(k)=h_{i}$ for $k=1,2, \cdots, L$. Thus, $\eta_{i}$ given by (7) can be rewritten as:

$$
\eta_{i}=\left|h_{i}\right|^{2} \frac{E_{s}}{\sigma_{n}^{2}}=\left|h_{i}\right|^{2} \eta_{s}
$$

where $\eta_{s}=\frac{E_{s}}{\sigma_{n}^{2}}$ is the local average SNR measured at the secondary receiver. It is thus note-worthy that the local average SNR is $L$ times more than the average SNR measured at the energy detector output, which can be expressed as $\eta_{0}=\frac{E_{s}}{L \sigma_{n}^{2}}$.

Under Rayleigh fading, the instantaneous SNR $\eta_{i}$ can be viewed as exponentially distributed

$$
f_{\eta_{i}}\left(\eta_{i}\right)=\frac{1}{\eta_{s}} e^{-\eta_{i} / \eta_{s}}
$$

For ease of calculation, we make the following approximation for equation (11) as

$$
P_{m i}\left(\eta_{i}\right) \approx \mathcal{Q}\left(\frac{\eta_{i}-\sqrt{L} \mathcal{Q}^{-1}\left(P_{f}\right)}{\sqrt{L+2 \eta_{s}}}\right)
$$


In what follows we shall define, for the sake of conciseness

$$
A=\frac{1}{\sqrt{L+2 \eta_{s}}}, B=\frac{\sqrt{L} \mathcal{Q}^{-1}\left(P_{f}\right)}{\sqrt{L+2 \eta_{s}}}
$$

then

$$
P_{m i}\left(\eta_{i}\right) \approx \mathcal{Q}\left(A \eta_{i}-B\right)
$$

Consequently, the average $P_{m}$ in the case of $\mathcal{M}_{1}, \bar{P}_{m}$, can be calculated by integrating (15) over (13) and making the change of variable $x=A \eta-B$ yielding

$$
\begin{aligned}
\bar{P}_{m} & =\int_{0}^{\infty} P_{m}\left(\eta_{i}\right) f_{\eta_{i}}\left(\eta_{i}\right) d \eta_{i} \\
& \approx \frac{1}{\eta_{s}} \int_{0}^{\infty} \mathcal{Q}\left(A \eta_{i}-B\right) e^{-\eta_{i} / \eta_{s}} d \eta_{i} \\
& =\frac{1}{A \eta_{s}} \int_{-B}^{\infty} \mathcal{Q}(x) e^{-\frac{x+B}{A \eta_{s}}} d x
\end{aligned}
$$

By capitalizing on the integrating characters of $\mathcal{Q}$-function [19], and after some simplification, we can derive the following formula for $\bar{P}_{m}$

$$
\bar{P}_{m} \approx \mathcal{Q}(-B)-e^{-\frac{B}{A \eta_{s}}} e^{\frac{1}{2 A^{2} \eta_{s}^{2}}} \mathcal{Q}\left(\frac{1}{A \eta_{s}}-B\right)
$$

In the case of $\mathcal{M}_{2}$, in order to obtain $\bar{P}_{m}$ in closed form, it desirable to obtain the PDF of $\eta_{i}$ given in (7). Unfortunately, So far as is known, there is no closed-form expression available for such a distribution. Therefore, it is computationally infeasible to obtain a closed-form expression for $\bar{P}_{m}$ in the present of temporal fading correlation. Instead of deriving a closed-form for $\bar{P}_{m}$, we estimate $\bar{P}_{m}$ through a Monte Carlo simulation.

Highly mobility of vehicles lead to an increase in Doppler shift $F_{d} T_{s}$, and thereby resulting in degraded correlation among sampling observations. In the case of $\mathcal{M}_{3}$, the sampling observations of the fading channel $\left\{h_{i}(k)\right\}$ are completely independent of each other, $\eta_{i}$ given by (7) can be reduced to the following equation

$$
\eta_{i}=\frac{E_{s}}{\sigma_{n}^{2}}
$$

It is plain to see that $\eta_{i}$ is independent of instantaneous fading statistics. Thus, the $\bar{P}_{m}$ in this case can be computed directly by (10) and (18).

\section{Cooperative Sensing with Counting Rule}

The concept of cooperative spectrum sensing is to utilize multiple SVUs at different locations and fuse their independent sensing messages into one unified decision inferring the presence or absence of the PU. In this section, we will ponder this approach based on hard fusion, and also look at the impact of the reporting channel fading to the global detection performance.

\subsection{Equivalent Local Probability of False Alarm and Miss Detection}

In consideration of the unreliable characteristic of the reporting channels, let us determine its effect on the reliability of the decision made by the FC. Let $v_{i}$ denote the decoded version of $z_{i}$ for the $i$ th SVU at the FC, the decoded rule can be expressed as

$$
v_{i}=\left\{\begin{array}{lll}
1 & \text { if } & z_{i} \geq 0 \\
0 & \text { if } & z_{i}<0
\end{array}\right.
$$


And, more remarkable, under the hypothesis $\mathcal{H}_{j}(j=0,1)$

$$
\begin{aligned}
\mathrm{E}\left[v_{i} \mid \mathcal{H}_{j}\right] & =P\left(v_{i}=1 \mid \mathcal{H}_{j}\right) \times 1+P\left(v_{i}=0 \mid \mathcal{H}_{j}\right) \times 0 \\
= & P\left(z_{i} \geq 0 \mid \mathcal{H}_{j}\right) \\
\mathrm{D}\left[v_{i} \mid \mathcal{H}_{j}\right] & =\mathrm{E}\left[v_{i}^{2} \mid \mathcal{H}_{j}\right]-\mathrm{E}\left[v_{i} \mid \mathcal{H}_{j}\right]^{2} \\
& =P\left(z_{i} \geq 0 \mid \mathcal{H}_{j}\right)-\left(P\left(z_{i} \geq 0 \mid \mathcal{H}_{j}\right)\right)^{2}
\end{aligned}
$$

where $\mathrm{E}[x], \mathrm{D}[x]$ denotes the expectation and variance operator with respect to $x$.

As can be seen from (20) and (21), the expectation and variance of received decision $v_{i}$ are directly depend on the probability $P\left(z_{i} \geq 0 \mid \mathcal{H}_{j}\right)$, which can also be referred to the equivalent probability of false-alarm and mis-detection of local sensing. For notational convenience, let us denote this probability by $P_{F i}$ and $P_{D i}$ under both hypothesis. In order to capture the statistical properties of $v_{i}$, we first need to obtain the probabilities $P_{F i}=f\left(z_{i} \geq 0 \mid \mathcal{H}_{1}\right)$ and $P_{D i}=f\left(z_{i} \geq 0 \mid \mathcal{H}_{0}\right)$.

Clearly, under the hypothesis $\mathcal{H}_{0}\left(\mathcal{H}_{1}\right), u_{i}$ obeys the following distribution with the parameter of $P_{f i}\left(P_{d i}\right)$.

$$
\begin{array}{r}
P\left(u_{i} \mid \mathcal{H}_{0}\right)=\left\{\begin{array}{cc}
P_{f i} & u_{i}=+1 \\
1-P_{f i} & u_{i}=-1
\end{array}\right. \\
P\left(u_{i} \mid \mathcal{H}_{1}\right)=\left\{\begin{array}{cc}
1-P_{m i} & u_{i}=+1 \\
P_{m i} & u_{i}=-1
\end{array}\right.
\end{array}
$$

Then, under the hypothesis $\mathcal{H}_{j}$, the equivalent probability of false alarm and miss detection can be written as:

$$
\begin{aligned}
P_{F i}=f\left(z_{i} \geq 0 \mid \mathcal{H}_{0}\right) & =\sum_{u_{i} \in\{+1,-1\}} f\left(z_{i} \geq 0 \mid u_{i}\right) P\left(u_{i} \mid \mathcal{H}_{0}\right) \\
& =f\left(z_{i} \geq 0 \mid u_{i}=+1\right) P_{f i}+f\left(z_{i} \geq 0 \mid u_{i}=-1\right)\left(1-P_{f i}\right) \\
P_{D i}=f\left(z_{i} \geq 0 \mid \mathcal{H}_{1}\right) & =\sum_{u_{i} \in\{+1,-1\}} f\left(z_{i} \geq 0 \mid u_{i}\right) P\left(u_{i} \mid \mathcal{H}_{1}\right) \\
& =f\left(z_{i} \geq 0 \mid u_{i}=+1\right) P_{d i}+f\left(z_{i} \geq 0 \mid u_{i}=-1\right)\left(1-P_{d i}\right)
\end{aligned}
$$

Without loss of generality, suppose that the reporting channels between SVUs and FC is also Rayleigh fading channel draw from CSCG distribution $\mathcal{C N}(0,2)$. In the other words, the PDF of the channel gain $g_{i}$ can be represented as

$$
f\left(g_{i}\right)=\left\{\begin{array}{cl}
g_{i} \exp \left(-\frac{g_{i}^{2}}{2}\right) & g_{i} \geq 0 \\
0 & g_{i}<0
\end{array}\right.
$$

On the basis of the fact that $y_{i}=g_{i} u_{i}, z_{i}=y_{i}+w_{i}$ and $w_{i}$ is a zero-mean Gaussian variable with variance $\delta_{i}^{2}$, we can easily get

$$
\begin{array}{r}
f\left(y_{i} \mid u_{i}\right)=\left\{\begin{array}{rr}
u_{i} y_{i} \exp \left(-\frac{y_{i}^{2}}{2}\right) & y_{i} \geq 0 \\
0 & y_{i}<0
\end{array}\right. \\
f\left(z_{i} \mid y_{i}\right)=\frac{1}{\sqrt{2 \pi} \delta_{i}} \exp \left(-\frac{\left(z_{i}-y_{i}\right)^{2}}{2 \delta_{i}^{2}}\right)
\end{array}
$$


Further still, according to this fact that $f\left(z_{i} \mid y_{i}, u_{i}\right)=f\left(z_{i} \mid y_{i}\right)$, which is stemmed from the fact that $\left(h_{i}, u_{i}\right) \rightarrow y_{i} \rightarrow z_{i}$ is a Markov chain, we can safely come to the following result

$$
\begin{aligned}
f\left(z_{i} \mid u_{i}\right) & =\frac{f\left(z_{i}, u_{i}\right)}{p\left(u_{i}\right)}=\frac{\int f\left(z_{i} \mid y_{i}, u_{i}\right) f\left(y_{i} \mid u_{i}\right) p\left(u_{i}\right) d y_{i}}{p\left(u_{i}\right)} \\
& =\int f\left(z_{i} \mid y_{i}\right) f\left(y_{i} \mid u_{i}\right) d y_{i}
\end{aligned}
$$

Then, substituting (25) and (26) into (27), we can obtain the conditional PDF of the received observation $z_{i}$, given the local decision $u_{i}=+1$, as

$$
\begin{aligned}
f\left(z_{i} \mid u_{i}=+1\right) & =\int_{0}^{\infty} \frac{1}{\sqrt{2 \pi} \delta_{i}} \exp \left(-\frac{\left(z_{i}-y_{i}\right)^{2}}{2 \delta_{i}^{2}}\right) y_{i} \exp \left(-\frac{y_{i}^{2}}{2}\right) d y_{i} \\
& =\frac{1}{\sqrt{2 \pi} \delta_{i}} \exp \left(-\frac{z_{i}^{2}}{2\left(1+\delta_{i}^{2}\right)}\right) \int_{0}^{\infty} y_{i} \exp \left(-\frac{\left(y_{i}-\frac{z_{i}}{1+\delta_{i}^{2}}\right)^{2}}{\frac{2 \delta_{i}^{2}}{1+\delta_{i}^{2}}}\right) d y_{i}
\end{aligned}
$$

More specifically performing a change of variable $t=y_{i}-\frac{z_{i}}{1+\delta_{i}^{2}}$ and making the best of the integral properties of $\mathcal{Q}$-function, we can obtain

$$
f\left(z_{i} \mid u_{i}=+1\right)=\frac{C^{2} \delta_{i}^{3}}{\sqrt{2 \pi}} \exp \left(-\frac{z_{i}^{2}}{2 \delta_{i}^{2}}\right)\left[1+\sqrt{2 \pi} C z_{i} \exp \left(\frac{C^{2} z_{i}^{2}}{2}\right) \mathcal{Q}\left(-C z_{i}\right)\right]
$$

$192 \quad$ where $C=\frac{1}{\delta_{i} \sqrt{1+\delta_{i}^{2}}}, \mathcal{Q}(-x)=1-\mathcal{Q}(x)$.

Integrating both sides of (29) with respect to $z_{k}$, we can obtain

$$
\begin{aligned}
& P\left(z_{i} \geq 0 \mid u_{i}=+1\right)=\int_{0}^{\infty} f\left(z_{i} \mid u_{i}=+1\right) d z_{i} \\
& =\frac{C^{2} \delta_{i}^{3}}{\sqrt{2 \pi}} \int_{0}^{\infty}\left[\exp \left(-\frac{z_{i}^{2}}{2 \delta_{i}^{2}}\right)+\frac{\sqrt{2 \pi}}{\delta_{i} \sqrt{1+\delta_{i}^{2}}} z_{i} \exp \left(-\frac{z_{i}^{2}}{2\left(1+\delta_{i}^{2}\right)}\right) \mathcal{Q}\left(-\frac{z_{i}}{\delta_{i} \sqrt{1+\delta_{i}^{2}}}\right)\right] d z_{i} \\
& =\frac{\delta_{i}}{\sqrt{2 \pi}\left(1+\delta_{i}^{2}\right)}\left[\frac{\sqrt{2 \pi} \delta_{i}}{2}+\frac{\sqrt{2 \pi} \sqrt{1+\delta_{i}^{2}}}{2 \delta_{i}}+\frac{\sqrt{2 \pi}}{2 \delta_{i}}\right] \\
& =\frac{1}{2}+\frac{1}{2 \sqrt{1+\delta_{i}^{2}}}=\frac{1}{2}\left(1+\sqrt{\frac{\gamma_{i}}{2+\gamma_{i}}}\right)
\end{aligned}
$$

193 where $\gamma_{i}$ denotes the SNR of the Rayleigh fading reporting channel.

A similar analysis can be conducted for the case of $u_{i}=-1$, we have

$$
\begin{gathered}
f\left(z_{i} \mid u_{i}=-1\right)=\frac{C^{2} \delta_{i}^{3}}{\sqrt{2 \pi}} \exp \left(-\frac{z_{i}^{2}}{2 \delta_{i}^{2}}\right)\left[1-\sqrt{2 \pi} C z_{i} \exp \left(\frac{C^{2} z_{i}^{2}}{2}\right) \mathcal{Q}\left(C z_{i}\right)\right] \\
P\left(z_{i} \geq 0 \mid u_{i}=-1\right)=\int_{0}^{\infty} f\left(z_{i} \mid u_{i}=-1\right) d z_{i}=\frac{1}{2}\left(1-\sqrt{\frac{\gamma_{i}}{2+\gamma_{i}}}\right)
\end{gathered}
$$


By substituting (30) and (32) in (22) and (23), and after some simplified calculations, we can obtain the equivalent local probability of false alarm and detection as

$$
\begin{aligned}
& P_{F i}=P\left(z_{i} \geq 0 \mid \mathcal{H}_{0}\right)=\frac{1}{2}+\left(P_{f i}-\frac{1}{2}\right) \sqrt{\frac{\gamma_{i}}{2+\gamma_{i}}} \\
& P_{D i}=P\left(z_{i} \geq 0 \mid \mathcal{H}_{1}\right)=\frac{1}{2}+\left(P_{d i}-\frac{1}{2}\right) \sqrt{\frac{\gamma_{i}}{2+\gamma_{i}}}
\end{aligned}
$$

and

$$
P_{M i}=1-P_{D i}=\frac{1}{2}+\left(P_{m i}-\frac{1}{2}\right) \sqrt{\frac{\gamma_{i}}{2+\gamma_{i}}}
$$

From (33) and (34), we can see that, if $P_{d i}>\frac{1}{2}$, then $P_{D i}<P_{d i}$. Only when $P_{d i}<\frac{1}{2}$, then $P_{D i}>P_{d i}$. In other words, the equivalent probability of miss detection at the FC high above the local probability of miss detection at each SUV. In the same manner, when $P_{f i}>\frac{1}{2}, P_{F i}<P_{f i}$. Only when $P_{f i}<\frac{1}{2}$, the channel error "increases" $P_{F i}$ to be higher than $P_{f i}$. Without doubt, this is achieved with an accompanied rise in the probability of miss detection.

Besides, we also see that, as the reporting channel becomes more unreliable ( low SNR situation, i.e., SNR $\left.\gamma_{i} \rightarrow-\infty\right)$, the equivalent probability $P_{D i}\left(P_{F i}\right)$ is close to $\frac{1}{2}$. As SNR $\gamma_{i} \rightarrow \infty, P_{D i}\left(P_{F i}\right)$ comes near to $P_{d i}\left(P_{f i}\right)$, I would like to say this is a perfect reporting channel.

\subsection{Global Probability of False Alarm and Miss Detection}

Assuming that the reporting channels do not interfere with each other, and the delay is negligible. Once the fusion center decodes $\left\{z_{i}=g_{i} u_{i}+w_{i} ; i=1,2, \cdots, N\right\}$ and gets $\left\{v_{i} ; i=1,2, \cdots, N\right\}$, a global test statistic based on the counting rule is calculated linearly as follows:

$$
\Lambda=\sum_{i=1}^{N} v_{i} \begin{cases}\geq T & \mathcal{H}_{1} \\ <T & \mathcal{H}_{0}\end{cases}
$$

where $T$ is the global decision threshold of counting rule, which can be in the form of $T=\alpha N$. This means that $T$ or more SVUs decide hypothesis $\mathcal{H}_{1}$, then the global decision is $\mathcal{H}_{1}$

From the preceding analysis, we can see that $v_{i}$ is a binary random variable which follows a Bernoulli distribution characterized by the associated $P_{F i}$ and $P_{D i}$. It is noteworthy that the pairs $\left(P_{F i}, P_{D i}\right)$ of different SVUs are not really the same because the pairs $\left(P_{f i}, P_{d i}\right)$ or $\gamma_{i}$ are different. So $\left\{v_{i}, i=1,2, \cdots, N\right\}$ is a set of independent and non-identically distributed random variables. In consequence, their sum $\Lambda=\sum_{i=1}^{N} v_{i}$ will not approach the Binomial distribution any more. This makes the theoretical analysis and evaluation of $\bar{Q}_{m}$ more sophisticated since it is indeed arduous to obtain a closed-form expression for such a distribution. Therefore, instead of relying on the exact distribution of $\Lambda$, we exploit a large-sample gaussian approximation for the sum of independent but not identically distributed random variables, known as the Lindberg-Feller CLT [20].

Theorem 1. Lindberg-Feller Central Limit Theorem (LF-CLT)

Assume that $\left\{X_{i}, i=1,2, \cdots, N\right\}$ is a set of independently and non-identically distributed random variables with mean $\mathrm{E}\left[X_{i}\right]=\mu_{i}$ and variance $\mathrm{D}\left[X_{i}\right]=\delta_{i}^{2}$. Further, assume that the two following regularity conditions are satisfied

$$
\mathrm{D}\left[X_{i}\right]>\beta_{1}
$$


and

$$
\mathrm{E}\left[\left|X_{i}-\mathrm{E}\left[X_{i}\right]\right|^{3}\right]<\beta_{2}
$$

where $\beta_{1}$ and $\beta_{2}$ are positive values. Then, for sufficiently big $N, \sum_{i=1}^{N} X_{i}$ converges asymptotically to a Gaussian distribution characterized by

$$
\sum_{i=1}^{N} X_{i} \rightarrow \mathcal{N}\left(\sum_{i=1}^{N} \mu_{i}, \quad \sum_{i=1}^{N} \delta_{i}^{2}\right)
$$

For the applicability of LF-CLT, we will show how above-mentioned Lindberg-Feller conditions are satisfied in the Appendix. Consequently, in a large cognitive vehicular network, the LF-CLT can be used to approximately describe the distribution of the global decision statistic under both hypothesis $\mathcal{H}_{0}$ and $\mathcal{H}_{1}$.

Because $\left\{v_{i}, i=1,2, \cdots, N\right\}$ are all independent of each other, when $N$ is sufficient large, in light of the LF-CLT [20], $\Lambda$ is asymptotically Gaussian distributed with mean

$$
\mu=\mathrm{E}\{\Lambda\}=\sum_{i=1}^{N} \mathrm{E}\left\{v_{i}\right\}=\left\{\begin{array}{cc}
\sum_{i=1}^{N} P_{F i} & \mathcal{H}_{0} \\
\sum_{i=1}^{N} P_{D i} & \mathcal{H}_{1}
\end{array}\right.
$$

and variance

$$
\sigma^{2}=\mathrm{D}\{\Lambda\}=\sum_{i=1}^{N} \mathrm{D}\left\{v_{i}\right\}=\left\{\begin{array}{cc}
\sum_{i=1}^{N} P_{F i}\left(1-P_{F i}\right) & \mathcal{H}_{0} \\
\sum_{i=1}^{N} P_{D i}\left(1-P_{D i}\right) & \mathcal{H}_{1}
\end{array}\right.
$$

Consequently, the global probability of false alarm and miss detection at the FC in this case can be described by

$$
\begin{gathered}
Q_{f}=\mathcal{Q}\left(\frac{T-\bar{\mu}}{\sqrt{\bar{\sigma}^{2}}}\right)=Q\left(\frac{T-\sum_{i=1}^{N} P_{F i}}{\sqrt{\sum_{i=1}^{N} P_{F i}\left(1-P_{F i}\right)}}\right) \\
Q_{m}=\mathcal{Q}\left(\frac{\bar{\mu}-T}{\sqrt{\bar{\sigma}^{2}}}\right)=\mathcal{Q}\left(\frac{\sum_{i=1}^{N} P_{D i}-T}{\sqrt{\sum_{i=1}^{N} P_{D i}\left(1-P_{D i}\right)}}\right)
\end{gathered}
$$

When the prior probabilities of presence and absence of PU are equal, i.e., $P\left(\mathcal{H}_{1}\right)=P\left(\mathcal{H}_{0}\right)=\frac{1}{2}$, the total probability of error detection can be written as

$$
\begin{aligned}
Q_{e} & =\mathcal{Q}_{f}+\mathcal{Q}_{m} \\
& =\mathcal{Q}\left(\frac{T-\sum_{i=1}^{N} P_{F i}}{\sum_{i=1}^{N} P_{F i}\left(1-P_{F i}\right)}\right)+\mathcal{Q}\left(\frac{\sum_{i=1}^{N} P_{D i}-T}{\sum_{i=1}^{N} P_{D i}\left(1-P_{D i}\right)}\right)
\end{aligned}
$$

\section{Numerical and Simulation Results}

In this section, both theoretical and simulated results are provided to illustrate spectrum sensing performance in cognitive vehicular networks. The PU signal is assumed to enjoy unit power and be any kind of modulated signal with carrier frequency of $900 \mathrm{Mhz}$ and bandwidth of $6 \mathrm{MHz}$. The sampling frequency in energy detector is equal to the bandwidth of PU signals, at the same time, the number of sampling observations during a sensing interval is $L=50$. For all simulation cases, the average SNR is assumed to be the same for all SVUs, e.g., $\eta_{0}=-8 \mathrm{~dB}$ and $\gamma_{0}=5.5 \mathrm{~dB}$. The performances with respect to the local (global) probability of miss detection is estimated to meet the constraint on the 
local (global) probability of false alarm of $P_{f}\left(Q_{f}\right)=0.1$. The number of realizations made to compute the simulating probability of miss detection is 10000, which are set to guarantee the simulation results can validate the theoretic analysis in the low probability region.

Firstly, we manifest the performance of spectrum sensing with energy detector in non-cooperative cases, which is a key springboard of the study in cooperative cases. In Fig. 4, we provide the curves of the local sensing performance based on energy detection as a function of Doppler shift $F_{d} T_{s}$. With the carrier frequency and the sampling interval fixed, $F_{d} T_{s}$ value goes up as the vehicle velocity $v$. It is illustrated that the probability of miss detection declines with the increase of vehicle velocity. This reason is that high mobility gives us an opportunity to achieve more received signal information, thereby enables SVUs to obtain higher sensing performance.

In Fig. 5 , we present the average $\bar{P}_{m}$ curves as a function of the average SNR of sensing channels. The capability of energy detector declines quickly with the reduce of the average received SNR from $10 \mathrm{~dB}$ to $-12 \mathrm{~dB}$. It can be seen that the simulating results are in accordance with the theoretical analysis for both $\mathcal{M}_{1}$ and $\mathcal{M}_{3}$, justifying the validity of the analysis. The figure indicates that the expression (17) and (11) (18) give the corresponding upper and lower bounds of the average $\bar{P}_{m}$ for spectrum sensing with energy detector under correlated Rayleigh fading channel.

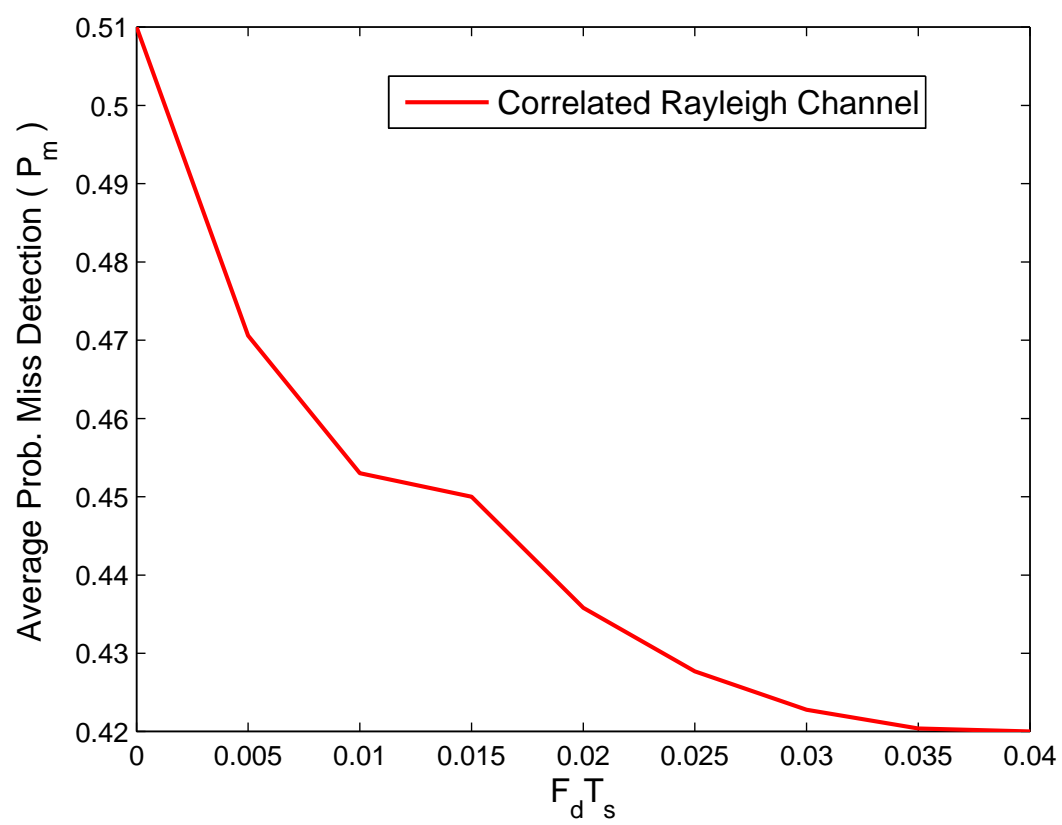

Figure 4. The local probability of miss detection for various $F_{d} T_{s}$. 


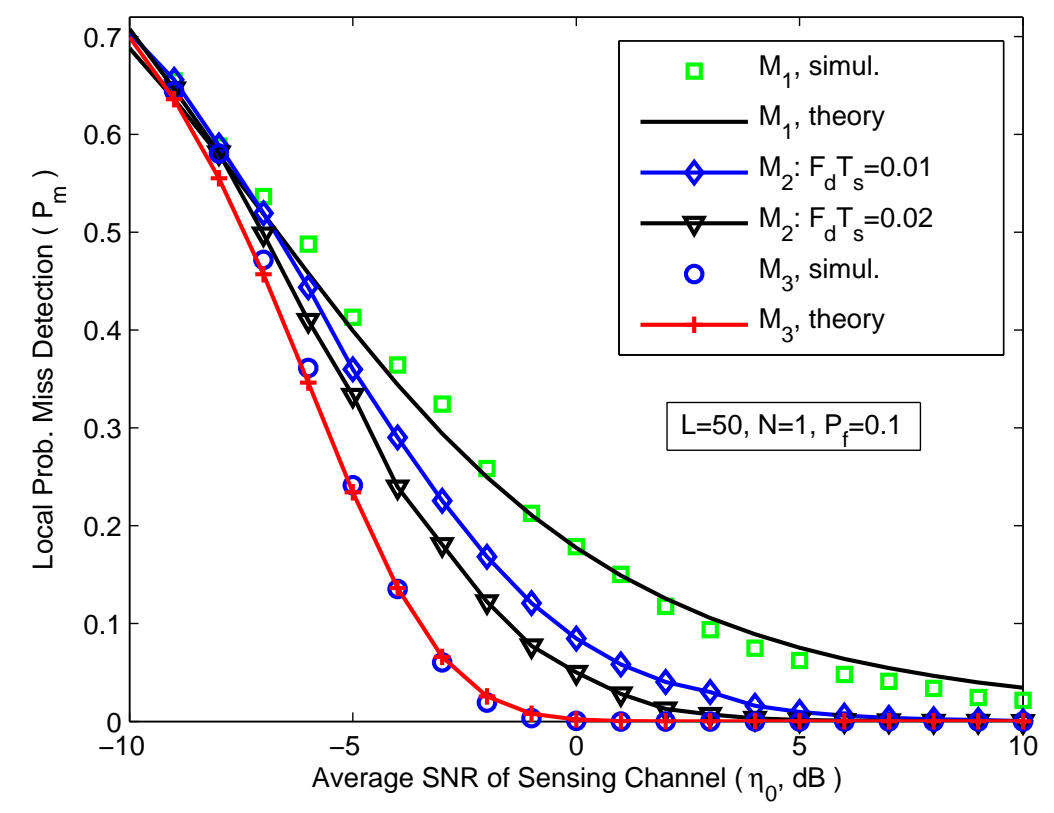

Figure 5. The local probability of miss detection for various average SNR of sensing channels.

Secondly, we focus on cooperative cases. In Fig. 6, we plot the global probability of miss detection $\left(Q_{m}\right)$ as a function of the number of SUVs for hard fusion based on counting rule. As expected, the sensing performance shows an upward trend along with the increase of the number of SUVs. When $N$ is extremely big, the global mis-detection probability approaches 0. For hard fusion, the Fig. 6 clearly demonstrate that the derived probability of miss detection based on Lindberg-Feller approximation is relatively poorer for a smaller number of SVUs. However, along with an increasing number of SVUs, the approximation asymptotically grows closer and closer to the exact sensing performance.

In Fig. 7, we present the ROC curves for different channel model. Note that, in the case of $\mathcal{M}_{1}$ and $\mathcal{M}_{3}$, we obtain the average mis-detection probability for local sensing, allowing for a much smoother global probability curve than the case of $\mathcal{M}_{2}$.

In Fig. 8, we present the average $Q_{m}$ curves as a function of the average SNR of reporting channels. In the condition of poor reporting SNR, large bit error occurs, which severely degrade the detection performance. We can clearly see that cooperative sensing performance improves quickly with the increase of the average SNR of reporting channel from $-8 \mathrm{~dB}$ to $8 \mathrm{~dB}$. Note that the sensing performance in AWGN channel is always better than in Reyleigh channel.

In Fig. 9, we plot the global probability of error detection $\left(Q_{e}\right)$ as a function of the local false-alarm probability. We can clearly see that, under certain conditions, there is an minimum probability of error detection, and that can be achieved when the local probabilities of false-alarm and mis-detection are equal $\left(P_{f}=P_{m}\right)$. In Fig. 10, we plot the minimum achievable probability of error detection $\left(Q_{e}\right)$ as a function of the number of SUVs. 


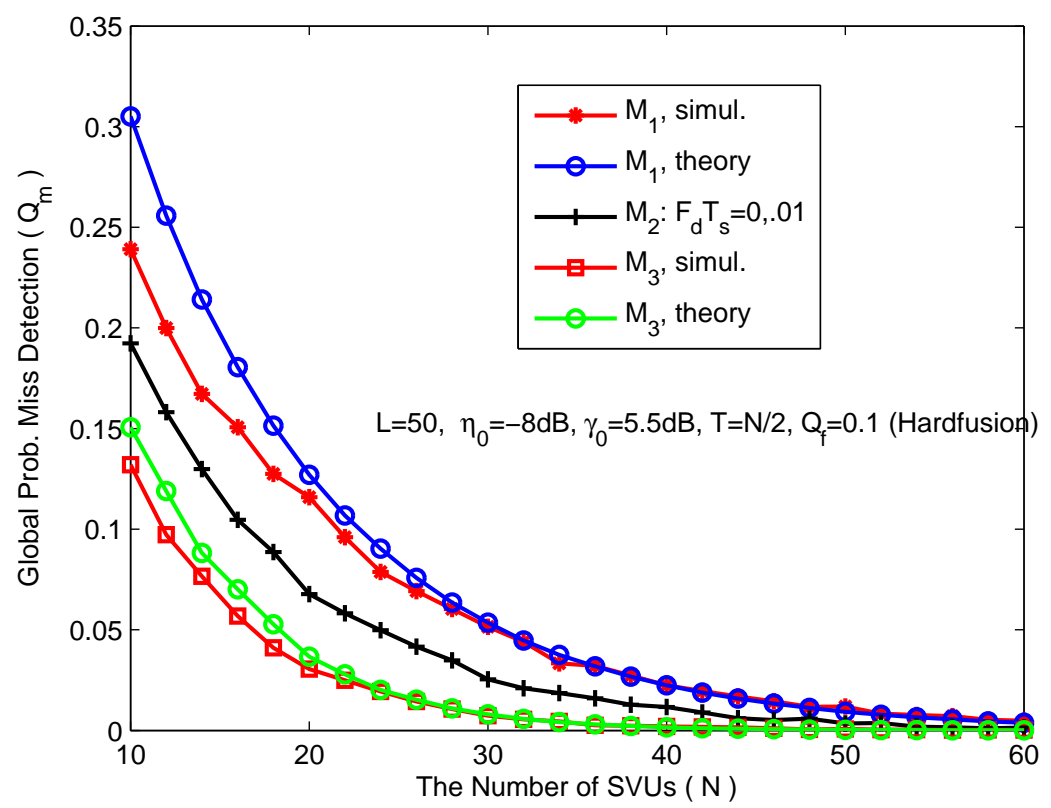

Figure 6. The global probability of miss detection for various number of SUVs.

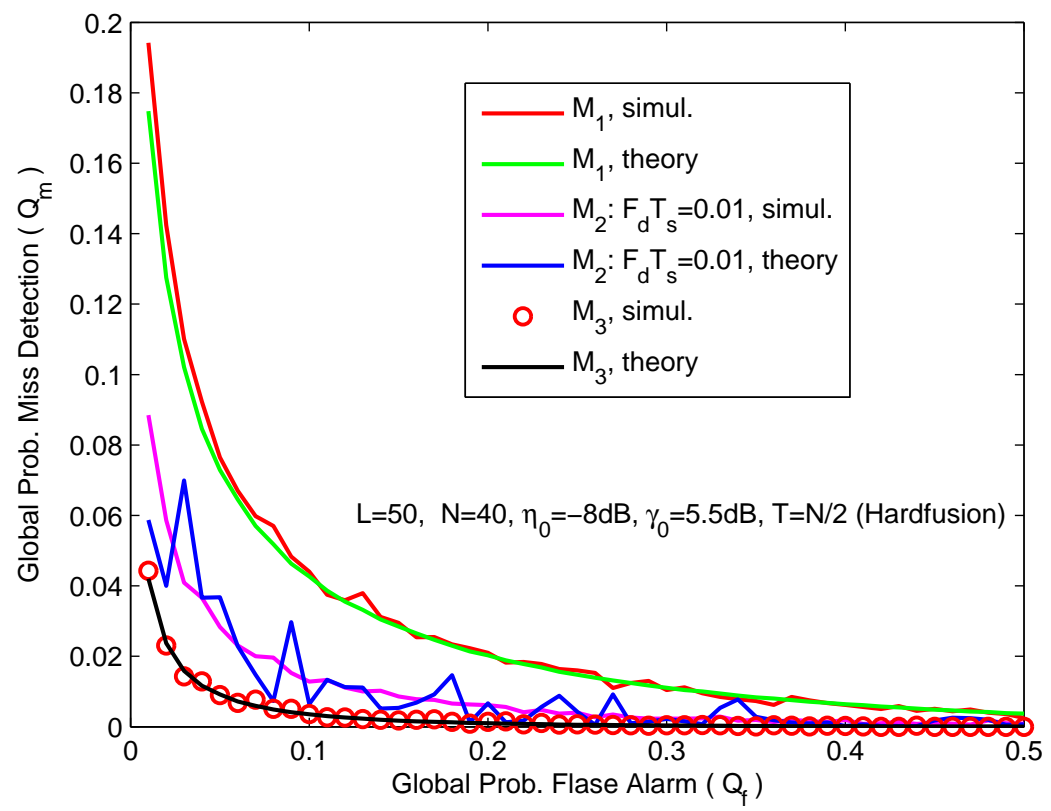

Figure 7. ROC curves for different channel model. 




Figure 8. The global probability of miss detection for various average SNR of reporting channels.

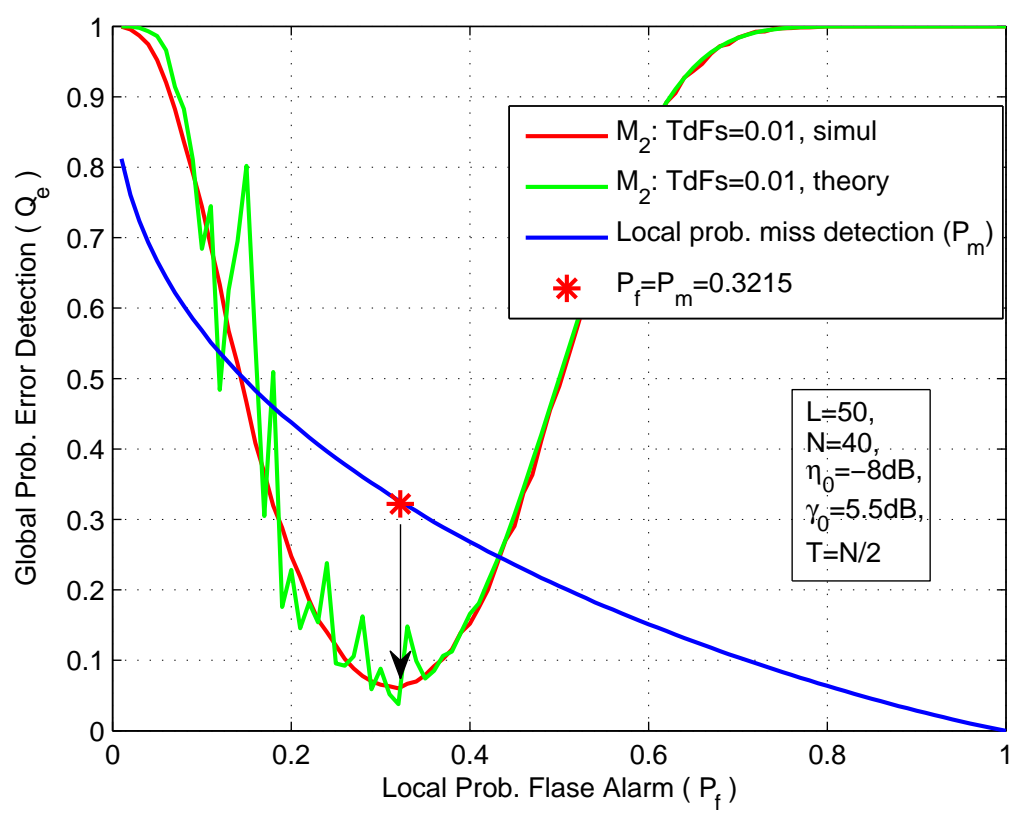

Figure 9. The global probability of error detection for various local probability of flase alarm. 


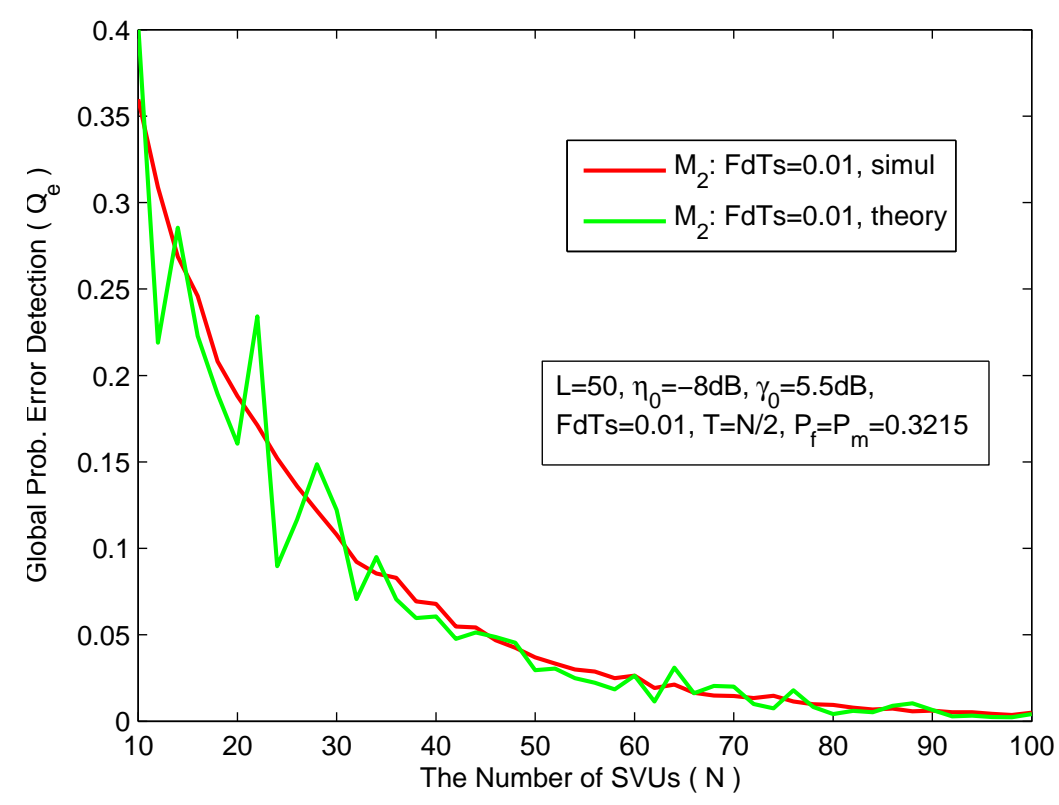

Figure 10. The global probability of error detection for various number of SVUs.

\section{Conclusion}

In this paper, we have researched the application of cognitive radio technique to vehicular environments for purpose of improving the reliability for vehicular communications. And for this, we evaluated the detection performance of spectrum sensing in mobile vehicular environments. Simulation results demonstrate that temporally correlation can not be neglected and be one of the considerable factors that may impact the sensing performance. In particular, highly mobility of the vehicles provides the opportunity to exploit temporal diversity at each vehicle. In spite of the fact that cooperative sensing have normally been considered as a viable means of producing better detection performance by making the most of spatial diversity among vehicles, we do believe that due to highly mobility, temporal diversity may be used in preference over vehicles' cooperation in the coming future.

Acknowledgments: This work was supported in part by the Key International Cooperation Project of Sichuan Province (2017HH0002), and in part by the NSFC under Grant 61601377 and Grant 61571373.

Author Contributions: Xiaomin Qian designed the system model, performed performance evaluations by theoretical analysis and simulation, and prepared the manuscript. Li Hao conceived the system model, carried out theoretical analysis and supervised the activities. Dadong Ni and Quang Thanh Tran discussed the main ideas and analyzed the results together. All authors approved the publication.

Conflicts of Interest: The authors declare no conflict of interest.

\section{Appendix}

In the following, we make clear how the two sufficient conditions (37) and (38) for the LF-CLT are satisfied for $\Lambda=\sum_{i=1}^{N} v_{i}$ under hypothesis $\mathcal{H}_{j}$, for $j=0,1$. Notice that in the LF-CLT, essentially, the objective of the two conditions is to ensure that no single random variable is dominate in its contribution to the summation operator. 
In the first step we shall prove that the first condition is satisfied. As can be seen from (21) and (34), the variance of $v_{i}$, for $k=1,2, \cdot, N$, under the hypotheses $\mathcal{H}_{1}$ can be represented as

$$
\begin{aligned}
D\left[v_{i} \mid \mathcal{H}_{1}\right] & =P_{D i}\left(1-P_{D i}\right) \\
& =\left[\frac{1}{2}+\left(P_{d i}-\frac{1}{2}\right) \sqrt{\frac{\gamma_{i}}{1+\gamma_{i}}}\right]\left[\frac{1}{2}-\left(P_{d i}-\frac{1}{2}\right) \sqrt{\frac{\gamma_{i}}{1+\gamma_{i}}}\right] \\
& =\frac{1}{4}-\left(P_{d i}-\frac{1}{2}\right)^{2}\left(\frac{\gamma_{i}}{1+\gamma_{i}}\right)
\end{aligned}
$$

It is sufficient to show that, under the hypothesis $\mathcal{H}_{1}$, the variance of $v_{i}$ is lower bounded by a positive value, as long as the $P_{d i}$ are bounded away from 0 and 1 .

In the next step, we show that the second condition (38) is satisfied. Let us make use of the fact that $v_{i}$ is a binary random variable which follows a Bernoulli distribution characterized by the associated $P_{F i}$ and $P_{D i}$. It is plain enough that under the hypotheses $\mathcal{H}_{1}$

$$
\begin{aligned}
\mathrm{E}\left[\left|v_{i}-\mathrm{E}\left[v_{i}\right]\right|^{3} \mid \mathcal{H}_{1}\right] & =P_{D i}\left(1-P_{D i}\right)\left(P_{D i}^{2}+\left(1-P_{D i}\right)^{2}\right) \\
& <P_{D i}\left(1-P_{D i}\right)
\end{aligned}
$$

Accordingly, it is straightforward to see that $P_{D i}\left(1-P_{D i}\right)$ is upper bounded by a positive value. In the above analysis, we prove that the two sufficient conditions (37) and (38) in the LF-CLT are satisfied under the assumption of hypothesis $\mathcal{H}_{1}$ explicitly, but the same proved method can be carried out to demonstrate the effectiveness under the hypothesis $\mathcal{H}_{0}$.

\section{References}

1. Mitola J.; Maguire, G. Q. Cognitive radio: Making software radios more personal. IEEE Pers. Commun. 1999, 6, 13-18.

2. Haykin, S. Cognitive radio: Brain-empowered wireless communications. IEEE J. Sel. Areas Commun. 2005, 23, 201-220.

3. Singh, K. D.; Rawat, P.; Bonnin, J. Cognitive radio for vehicular ad hoc networks (CR-VANETs): approaches and challenges. EURASIP J. wireless commun. net. 2014, 2014.1: 49.

4. Wang, X. Y.; Ho, P. H. A Novel Sensing Coordination Framework for CR-VANETs. IEEE Trans. Veh. Technol. 2010, 59, 1936-1948.

5. Ali, A.; Hamouda, W. Advances on Spectrum Sensing for Cognitive Radio Networks: Theory and Applications. IEEE Commun. surveys tut. 2017, 19, 1277-1304.

6. Cicho-, K.; Kliks, A.; Bogucka, H. Energy-efficient cooperative spectrum sensing: A survey. IEEE Commun. Surveys Tut. 2016, 18, 1861-1886.

7. Mumtaz, S.; Huq, K. M. S.; Ashraf, M. I.; Rodriguez, J.; Monteiro, V.; Politis, C. Cognitive Vehicular Communication for 5G. IEEE Commun. Maga. 2015, 53, 109-117.

8. Felice, M. D.; Chowdhury, K. R.; Bononi, L. Analyzing the potential of cooperative Cognitive Radio technology on inter-vehicle communication. In Proceedings of the IFIP Wireless Days, Venice, Italy, 1-6, Oct. 2010.

9. Rasheed, H.; Rajatheva, N. Spectrum sensing for cognitive vehicular networks over composite fading. Int. J. Veh. Technol., 2011, 2011, 1-9.

10. Liu, Y.; Xie, S.; Yu, R.; Zhang, Y.; Zhang, X.; Yuen, C. Exploiting temporal and spatial diversities for spectrum sensing and access in cognitive vehicular net- works. Wireless Commun. Mobile Comput. 2015, 15, 2079-2094.

11. Aygun, B.; Wyglinski, A. M. A Voting Based Distributed Cooperative Spectrum Sensing Strategy for Connected Vehicles. IEEE Trans. Veh. Technol., 2017, 66, 5109-5121.

12. Chembe, C., Noor, R. M., Ahmedy, I., Oche, M., Kunda, D., Liu, C. H. "Spectrum sensing in cognitive vehicular network: State-of-Art, challenges and open issues," Computer Commun. 2017, 97, 15-30.

13. Rahaman, M. F.; Khan, M. Z. Low Complexity Optimal Hard Decision Fusion under Neyman-Pearson Criterion. IEEE Signal Process. Lett. 2017, 1, 1-5. 
14. Chaudhari, S.; Lunden, J.; Koivunen, V.; Poor, H. V. Cooperative sensing with imperfect reporting channels: Hard decisions or soft decisions? IEEE Trans. Signal Process. 2012, 60, 18-28.

15. Simon, M. K.; Alouini, M. Digital communication over fading channels., Vol. 95, John Wiley \& Sons, 2005.

16. Tsitsiklis, J. N. Decentralized detection. Advances in Statistical Signal Processing. Greenwich, CT: JAI, 1993, 2, 297-344.

17. Herath, S. P.; Rajatheva, N.; Tellambura, C. Energy detection of unknown signals in fading and diversity reception. IEEE Trans. Commun., 2011, 59, 2443-2453.

18. Atapattu, S.; Tellambura, C.; Jiang, H. Energy detection based cooperative spectrum sensing in cognitive radio networks. IEEE Trans. wireless commun., 2011, 10, 1232-1241.

19. Nuttall, A. H. Some integrals involving the Q-function. DTIC Document, Tech.Rep., 1972.

20. Peebles, P. Z.; Shi, B. E. Probability, random variables, and random signal principles. McGraw-Hill: New York, USA, 2001. 